\title{
An Unusual Cause of Abdominal Pain
}

Myo Khine, ${ }^{1}$ MS (Malaya), MRCSEd, April Camilla Roslani, ${ }^{1}{ }_{M S}$ (Malaya), MRCSEd

A 36-year-old man presented with a 7-day history of intermittent right-sided abdominal pain. He also had a reducible right-sided inguinal hernia for the past 10 years. He was initially managed as acute hepatitis as there was right hypochondrial tenderness associated with elevated liver enzymes. Two days later, a mass was palpable in the right iliac fossa. A computed tomography (CT) scan of the abdomen was performed.

What does the CT scan show?

1) Appendiceal mass

2) Omental torsion

3) Empyema of the gallbladder

4) Retroperitoneal tumour

5) Ascending colon carcinoma

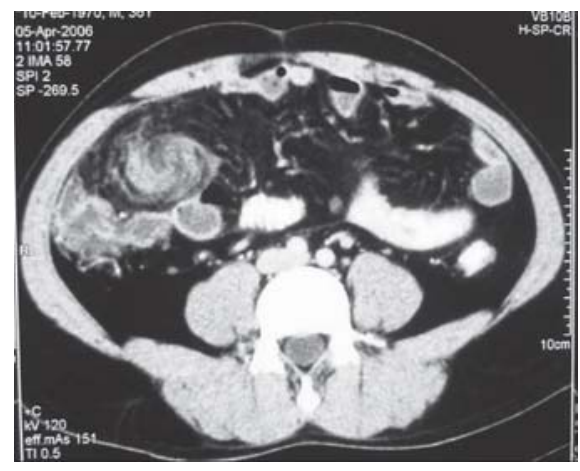

Fig. 1. CT scan of the abdomen, axial view, above the iliac crests.

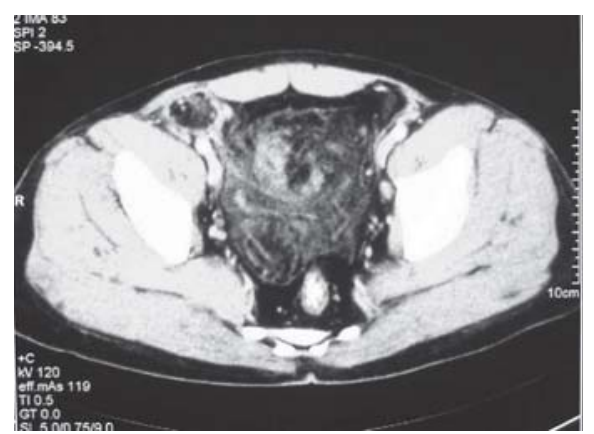

Fig. 2. CT scan of the pelvis, axial view, below the iliac crests.
Torsion of the greater omentum is an uncommon surgical emergency that was first described by Eitel in 1899. It may be classified as primary (idiopathic) or secondary. Predisposing factors to primary torsion are obesity and anatomical alterations or vascular anomalies of the omentum. Secondary torsion is more common, and is associated with omental (neoplastic lesion), parietal (inguinal hernia, adhesions) or visceral (appendicitis, cholecystitis, salpingitis) pathology. ${ }^{1}$

Precipitating factors for torsion are change of position, trauma and a sudden increase in intra-abdominal pressure. ${ }^{2}$ Initially, the omentum becomes congested due to obstruction of venous return, followed by arterial obstruction resulting in tissue necrosis, secondary infection and adhesion formation.

CT scans can demonstrate a characteristic "whirl" pattern, ${ }^{3}$ which is diagnostic, as in this case (Fig. 1). This CT also shows omentum in the right inguinal canal (Fig. 2), suggesting secondary omental torsion. These findings were confirmed at laparotomy.

However, CT is not always conclusive, and may only show an increase in fat density with hyper-attenuated streaks, deep to the parietal peritoneum of the anterior abdominal wall. Diagnosis is often only made intraoperatively.

Treatment is by surgical omentectomy, which can be performed open or laparoscopically. ${ }^{4}$

\section{REFERENCES}

1. Feo CF, Porcu A, Ginesu GC, Dettori G. Primary torsion of the greater omentum: a difficult diagnosis. Dig Dis Sci 2005;50:1283-4.

2. Hirano Y, Oyama K, Nozawa H, Hara T, Nakada K, Hada M, et al. Leftsided omental torsion with inguinal hernia. World J Gastroenterol 2006;12:662-4.

3. Itinteang $\mathrm{T}$, van Gelderen WF, Irwin RJ. Omental whirl: torsion of the greater omentum. Aust N Z J Surg 2004;74:702-4.

4. Gassner PE, Cox MR, Cregan PC. Torsion of the omentum: diagnosis and resection at laparoscopy. Aust N Z J Surg 1999;69:466-7.

\footnotetext{
${ }^{1}$ Department of Surgery, Faculty of Medicine, University of Malaya. Address for Correspondence: April Camilla Roslani, Department of Surgery, Faculty of Medicine, University of Malaya, 50603 Kuala Lumpur, Malaysia. Email: april@ummc.edu.my
}

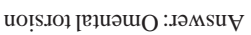

